\title{
Pretibial Myxedema: A case report
}

\author{
by Heather L. Salton, DPM ${ }^{1 ⿴}$
}

The Foot and Ankle Online Journal 3 (9): 3

A case report describing pretibial myxedema is presented. A 50 year-old African-American female presents with a painful pretibial, multinodular plaque. Additional nodules were present on the foot. Clinical presentation including skin biopsy and treatment is discussed. The pathogenesis of pretibial myxedema is unknown. Treatment consists of corticosteroids under occlusion and other treatments are being investigated.

Key words: Myxedema.

Published: September, 2010

This is an Open Access article distributed under the terms of the Creative Commons Attribution License. It permits unrestricted use, distribution, and reproduction in any medium, provided the original work is properly cited. @The Foot and Ankle Online Journal (www.faoj.org)

$\mathrm{P}$ retibial myxedema (PTM) is a term used for the dermal deposition of acid mucopolysaccharides (glycosaminoglycans). These acids are mainly composed of hyaluronic acid (HA) and deposit commonly on the lower legs, feet and particularly the shins. ${ }^{1,2}$

This clinical sign is most often associated with Graves disease, occurring in approximately $2-5 \%$ of these patients. ${ }^{1}$ It is commonly seen in combination with ophthalmopathy. Graves disease consists of four components including hyperthyroidism secondary to diffuse goiter, ophthalmopathy and dermopathy which is usually PTM, and the presence of long acting thyroid stimulator (LATS). ${ }^{3}$ It can and very rarely occurs in non-thyrotoxic thyroid states such as hypothyroidism and euthyroid disorders. These disorders present with three of the four components of Graves disease that include ophthalmopathy, dermopathy, and LATS. ${ }^{4}$

Pretibial myxedema presents as a nodular or plaquelike appearance of the skin in the pretibial area. It is often bilateral with a predilection for females in their forties and fifties. ${ }^{4,6}$

Address correspondence to: Heather L. Salton, DPM

${ }^{1}$ Private practice, Puyallup Foot and Ankle Center, Puyallup, Washington. St. Francis Hospital, Federal Way, Washington.
The dermopathy may also present with hyperpigmentation and/or non-pitting edema that may be caused by the hygroscopic property of HA., The skin also often has a peau d'orange (orange-skin peel) appearance caused by dilated, follicular openings.

\section{Case Presentation}

A 50 year-old African-American female presents with a five year history of nodular plaques involving the pretibial region of the left lower leg. (Fig.1A and B)

The nodules have progressively enlarged causing discomfort secondary to pressure from shoe gear. Her medical history was significant for diffuse goiter diagnosed in 1993 and breast cancer. She was being treated with Ultravate ${ }^{\circledR}$ and Synthroid $\AA$. At the time of PTM diagnosis, the patient was non-thyrotoxic and presented with a T-3 of 29 (22-35\%), free T-4 (FT4) of $1.2(0.8-1.8 \mathrm{ng} / \mathrm{dL})$, total $\mathrm{T}-4$ of 8.1 (4.5-12.5 $\mathrm{mcg} / \mathrm{dL}$ ), total free T-4 index of 2.3 (1.4-3.8) and a TSH with reflex to FT4 of 2.93 (0.4-5.5). 


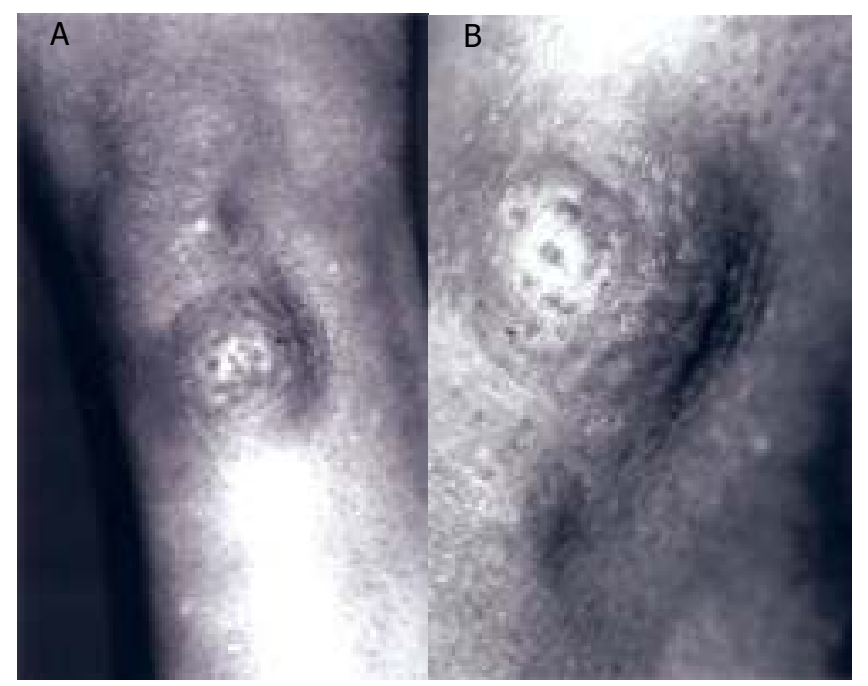

Figure 1 Well circumscribed, pretibial, nodular plaque with an orange-skin peel appearance. (A) Close-up photograph of the nodular plaque in the pretibial region of the lower leg. (B)

Physical examination revealed a well circumscribed, multi-nodular, pretibial plaque located on her left lower leg. Additional nodules were present on the dorsum of her left foot over the area of the cuboid and proximal to the first metatarsophalangeal joint.

\section{Histopathology}

A $4 \times 4 \times 3 \mathrm{~mm}$ punch biopsy revealed hyperkeratosis, epidermal hyperplasia and edema located throughout the papillary dermis. This stained positive for mucin with Alcian blue. Hematoxylin and eosin stain shows mucin accumulation causing separation and fraying of collagen fibrils. (Figs.2A and B)

\section{Discussion}

Pretibial myxedema is most accurately diagnosed with a skin biopsy. Characteristics include a hyperkeratotic epidermis and a dermis that contains increased amounts of mucin or glycosaminoglycans (GAG), mainly hyaluronate. These substances are produced by dermal fibroblasts and their synthesis is regulated by thyroid and glucocorticoid hormones. ${ }^{5}$

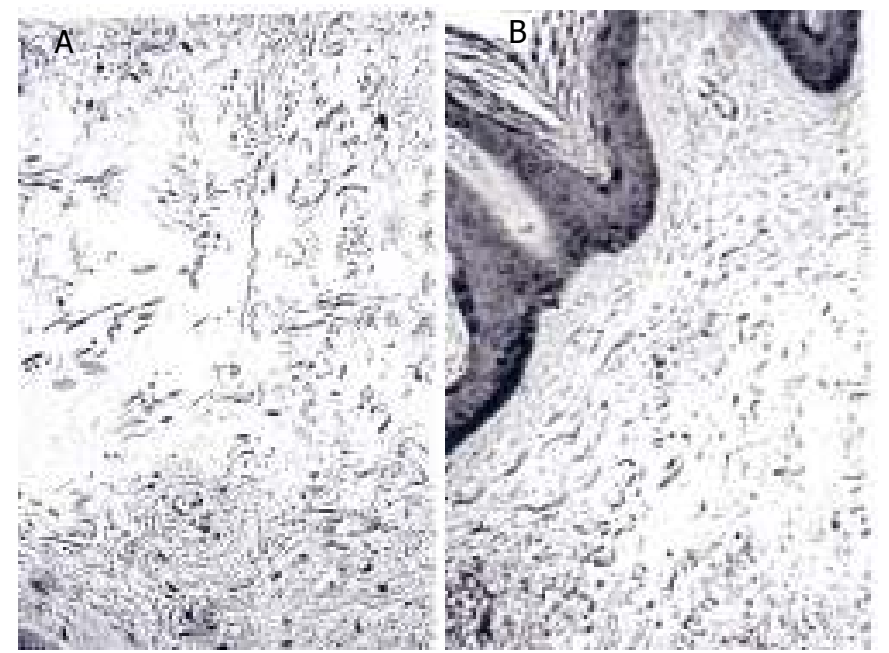

Figures 2 Forty times magnified Hematoxylin-Eosin stain of the dermis showing mucin accumulation. (A) Epidermis and dermal H\&E stain showing mucin accumulation causing separation and fraying of collagen fibrils. (B)

The hyaluronic acid accumulates extensively in the upper dermis. Stellate or spindle-shaped fibroblasts may show an increase in number along the middle to lower dermis. Separation and fraying of collagen fibrils is seen secondary to the large accumulation of GAG., ${ }^{4,5}$

The pathogenesis of PTM is still unknown. The presence of LATS is thought to play a role in the development, but not the severity of PTM. LATS is an immunoglobulin of the $\operatorname{IgG}$ family that represents an autoantibody to the TSH receptor. It is present in 95 to $100 \%$ of patients with PTM.

It is believed that this antibody with other anti-thyroid stimulator hormone-receptor antibodies can crossreact with the pretibial dermis to stimulate collagen synthesis by fibroblasts. However, a cofactor may be needed for this cross-reaction to occur as it has not been shown to directly affect fibroblasts from pretibial tissue in vitro. ${ }^{5,7}$

Serum-derived-hyaluronan-associated protein (SHAP) is also thought to play a role in the development of pretibial myxedema. SHAP has been characterized as a mediator between the cell surface and HA, and appears to be involved in both HA-cell interactions and HA metabolism. 
It has been isolated as an HA binding protein found on the surface of fibroblasts particularly in the middle to lower dermis indicating that $\mathrm{HA}$ accumulation progresses from the upper to the lower dermis. Therefore, the interaction between fibroblasts and SHAP may also result in the deposition of excess HA leading to cutaneous changes seen in pretibial myxedema. ${ }^{2}$

\section{Summary}

Although this patient was lost to follow-up and received no further treatment, the majority of cases do not require therapy with $50 \%$ of mild cases achieving complete remission after several years. Treatment primarily consists of palliative care with corticosteroids applied under occlusion. ${ }^{7}$ Other treatment methods are being studied in more severe cases. The use of intra-lesional octreotide, an antagonist to insulin-like growth factor 1 (IGF-1), suggests down regulation of hyaluronic acid production by lesional fibroblasts. ${ }^{8}$ The immunologic role suspected in the pathogenesis and immunomodulation with high dose intravenous immunoglobulin therapy is also being studied. ${ }^{\text {im }}$

\section{References}

1. Ishizawa T, Sugiki H, Anzai S, Kondo S. Pretibial myxedema with Graves' disease: A case report and review of Japanese literature. J Dermatol 1998; 25: 264-268.

2. Shishido M, Kuroda K, Tsukifuji R, Fujita M,. Shinkai H. A case of pretibial myxedema associated with Graves' disease: An immunohistochemical study of serum-derived hyaluronanassociated protein. J Dermatol 1995 22: 948-952.

3. Lynch PJ, Maize JC, Sisson JC. Pretibial myxedema and nonthyrotoxic thyroid disease. Arch Dermatol Jan 1973;107: 107-111.

4. Cannavo SP, Borgia F, Vaccaro M, Guarneri F, Magliolo E, Guarneri B. Pretibial myxoedema associated with Hashimoto's thyroiditis. J Eur Acad Dermatol Venereol 2002; 16: 625-257. 5. Sauer P, Brandes B, Mahmarian RR. Lower extremity manifestations of Graves' disease. J Foot Ankle Surg 1995 34(5): 489-497.

6. Kriss J. Pathogenesis and treatment of pretibial myxedema. Endo Metab Clinics N Amer 1987 16(2): 409-415.

7. Fatourechi V. Pretibial myxedema: pathophysiology and treatment options. Am J Clin Dermatol 2005 6(5):295-309.
8. Shinohara M, Hamasaki Y, Katayama I. Refractory pretibial myxoedema with response to intralesional insulin-like growth factor 1 antagonist (octreotide): downregulation of hyaluronic acid production by the lesional fibroblasts. Br J Dermatol 2000 143(5):1083-1086.

9. Jolles S, Hughes J. Use of IGIV in the treatment of atopic dermatitis, urticaria, scleromyxedema, pyoderma gangrenosum, psoriasis, and pretibial myxedema. Int Immunopharmacol 2006 6(4): 579-591. 